\title{
The digitalization of analogue stereo photographs and the creation of the digital stereo archive
}

\author{
Rodrigo Peixoto; Cicant - ULHT; Lisbon - Portugal \\ Filipe Costa Luz; HEI-Lab - ULHT; Lisbon - Portugal \\ Jorge Oliveira; HEI-Lab - ULHT; Lisbon - Portugal
}

\begin{abstract}
This paper analyzes the problems arising from the remediation of the relief effect in the transition from analog to digital of stereo photography. One of the main problems in this conversion is the portability of the awe effect that constitutes an important part of the experience when viewing a stereo pair. This image conversion process, necessary to the creation and dissemination of digital files of 19th century stereoscopic photography, is not linear. The digital stereoscopic projection cards present a number of difficulties for a proper consistency reproduction of the relief effect. Through the study comparison of different viewing apparatus (both digital and analogue including 3D and VR) of a specific stereo image, we will present important results achieved with a sample of 134 participants that were exposed to these devices and propose a guideline manual for the digital stereo archive.

For the developing of this work it has been crucial the research done by both authors for the Stereo Visual Culture project (supported by the FCT Foundation ref. PTDC / IVCCOM /5223/2012), the stereopsis analysis made with the research center HEI-Lab (Digital Human-Environment Interaction Lab) and through the particular case study based on the VR application developed for the recreation of XIX century Carlos Relvas' studio, in exhibition at Museu Nacional de Arte Contemporânea do Chiado (Lisbon) from November 2018 until February 2019.
\end{abstract}

Keywords: Stereoscopy; Displays; Museums; Digitalization; Archive;

\section{Introduction: brief historical notes}

In recent times we have witnessed a resurgence of stereoscopy as a technique associated with different imaging contexts. As an apparatus for transforming the visibility of technical images, whether they are still or moving images, its presence has grown in cinemas, museums, art galleries or academic presentations.

The protocol of vision that the stereoscopic photography inaugurates is a protocol of excess. This fascination provided by stereoscopic photography is based (remarkably, but not only) on its haptic particularity. The sensitive material that the world projects, and of which we are receptors, cannot be divided into isolated qualities. This division of our sensory heritage was an imposition of evolution, structuring our knowledge of the world in five main senses. But some senses remain still hostage to an earlier connection, such as the smell and taste. The haptic sense shares this connection by being constituted as tact and vision [1].

The general public quite often recognizes S3D films, anaglyph glasses or VR technologies and applications, however stereoscopic images and $19^{\text {th }}$ century stereo apparatus are commonly unknown. Surprise is the common reaction when museum visitants and our students interact with Brewster-type stereoscope (David Brewster, 1849) or Holmes viewer (Oliver
W. Holmes, 1861), reflecting the novelty of the stereopsis experience and the lack of knowledge about the quality and relief effect of

the XIX century stereo cards.

Charles Wheatstone started an important revolution when combined two slightly different perspective of the same drawing to reveal a three-dimensional image. His stereoscope (1838) had a relevant role for the advent of stereopsis vision studies and art experiences. David Brewster and Oliver W. Holmes push forward the stereo devices and with the contribution of the new emergent photography media, a new industry of stereo photography explode in Victorian period, more precisely after the great exhibition in Crystal Palace (1851). Photographers like Antoine Claudet, Thomas R. Williams, George Washington Wilson, Alfred Seaman, William England, Eadweard Muybridge, among others, all contributed for an important image archive of the world in XIX century and first quarter of XX century, but with the extraordinary experience of 3-D «which will make you gasp in wonder, their old sorcery still potent after all those years». [2]

The recent advances in VR can be looked upon as clear media hybridization. Modern head-mounted displays, such as Oculus Rift or HTC Vive, remediate victorian stereoscopic viewers in several ways. For instance, the idea of disembodiment present in both displays, both of them intend to create a realistic and immersive experience by transporting the viewer to the realm of the projected image.

When it happens for the first time, this experience it is always underlined by some astonishment expressions.

\section{Stereo photography digitalization - main concerns}

By imposing a visual prosthesis to its viewer (only then can he/she access the three-dimensional reality contained in the double flat image), the stereoscopic photography performs a double sensory action on the eye. The lenses of the viewer make possible for our eyes to focus the images (allowing their independent visualization and consequently threedimensionality), and work as a catalyst for detail. In stereo photographic images two instances of the visual spectacle coexist - detail and relief. The visualization of a stereo photography can be seen as a visual event that triggers the aweeffect and stimulates the haptic sense $[3,4]$.

What stereoscopy as an applied technique introduces is amazement in the act of seeing in itself. It enhances the immersive ability of photography, presenting a threedimensional space, and catalyzes haptic and visual sensation. This haptic sensation or awe-effect is one of our main concern transforming analogue images into digital files.

\section{The stereo archive}

How does this transformation alter or not the haptic sensation and consequently the seminal awe-effect of stereoscopic images by dematerializing the images (in projections), modifying the visibility device (through 
visualization in anaglyph) or increasing the level of immersion (imposing VR devices)? These questions stand as fundamental for this article.

With the emergence of relatively cheap and easy to use 3D TVs, new possibilities for stereo photography digital archives emerge. We all know the increase in ease of access and query that digital archives offer. We can advocate that the experience is not the same, that nothing replaces direct access to the original, but still these archives remain an essential tool in certain phases of the research process.

It seems to us that this is the time to constitute effective and functional digital stereo archives that allow the viewing of the stereo photographs in optimum conditions for a vast number of individuals throughout the globe. By defining the fundamental instructions for this process and the desirable outputs we can establish a standard for both the observer and the archivist.

With regard to the digital reconstitution of the awe-effect, this article seeks to understand what changes occur in this conversion. Through the comparison of different viewing apparatus (both digital and analogue including 3D and VR) and the inquiry of a considerable number of subjects exposed to them, we will propose a guideline manual for the digital stereo archive and viewing conditions of digitized analogue stereo photographs, carrying in mind our own experience as researchers in the photography field.

On the other hand, the digitization and dematerialization of analogue stereoscopic pairs also alters access to all the information contained in the materiality of the supports. From the indelible signature of photographic processes (impressions in albumin or bromide produce various colorations, grain and detail), the textual information contained in the cards or glasses (titles of images, texts or handwritten notes by the authors or owners of the images), or even the original packaging and its form of organization in volumes, boxes, or other device - the biography of the images. This level of material information is sometimes as important as visual image information to a researcher.

In order to overcome this aspect it is necessary to understand that in the case of stereoscopic photography it becomes crucial to allow access to the reproduction of the original support (card, glass or other) containing all the information outside the space of the photographic image; the original packaging; and the context (is it part of a larger collection or of an archive of an ethnographer, for example), in addition to all the information that is usually already included in the technical data sheets (author, process, date, etc.).

The importance of photography derives undoubtedly from its visual content but also from its existence as a material object. Although the visual content of a photographic image appears to be its most pressing manifestation, access to the information contained in the material object clarifies - confirms or denies- its visual content, creating a social and cultural context for the image. This materiality of the image elucidates about its life at the time it was created, establishing a connection with the ways in which it was seen by its contemporaries, and by doing so, helping us to understand their purpose.

A secondary aim of this investigation is to constitute important knowledge for the use of stereo devices in exhibition spaces such as museums and art galleries. Through our own personal experience, we found that there is an absence of studies that deal with the use of stereo devices in the context of exhibitions, whether artistic or historical, focusing on the observer experience.

\section{Study Concept}

Based on stereoscopic exhibitions made by the authors during 2015-2019 in four Portuguese museums, there were many questions that arise from the way stereoscopic photographs are presented to public. What is the reliability for digital conversion of analogue stereo archives? Is it possible to reproduce digitally the experience of interacting with a Holmes or Brewster viewer? What are the differences in viewer experience of a stereo image projected in anaglyph to a wall, a HD 3DTV screen, or a VR display? Should we retouch scratches and dust from the digitalized images for a more engaging interaction?

The museums exhibitions have different roles, from one side it's important to share knowledge and archives, but at the same time it's important for the institutions to guarantee the quality of each visitant experience. This is a main issue, because the embodiment of the visitants in museums it's certainly different when exposed to the physical interaction with stereoscopic viewers, digital or analogue. Both present very singular and individualized experiences.

Our senses enable and modify our experience with stereo devices. The same display, space or photograph stimulates different sensations to users, since in the human perceptual system the stereo sensation is not formed identical in each stereoscopic system. The body relation when interacting with a Verascope viewer is very different from standing in front of a $3 \mathrm{~d}$ projection or moving around the space wearing anaglyph glasses. Feeling the wooden model of a Holmes Viewer is distinctive from putting on a plastic VR helmet. The mixed senses of smell, touch, heard and see, magnify the experience of stereoscopic image into a different level,

So, this study aims to collect information from participants that could lead us to map the main emotional variances of each viewer when exposed to the five stereoscopic devices.

\section{Apparatus Display}

(In order to establish a better comparison between the different viewing apparatus we have decided to use the same image in all the devices.).

This section presents the different stereoscopic display setup used in this study. The experience took place in MovLab facilities, which allowed us the simultaneous observation of the users interactions with the devices. During 6 days in different timetables, groups of 15 students went to MovLab facilities to interact with the displays while supervised by a team of 5 observers (researches from the field of psychology, videogames and communication sciences).

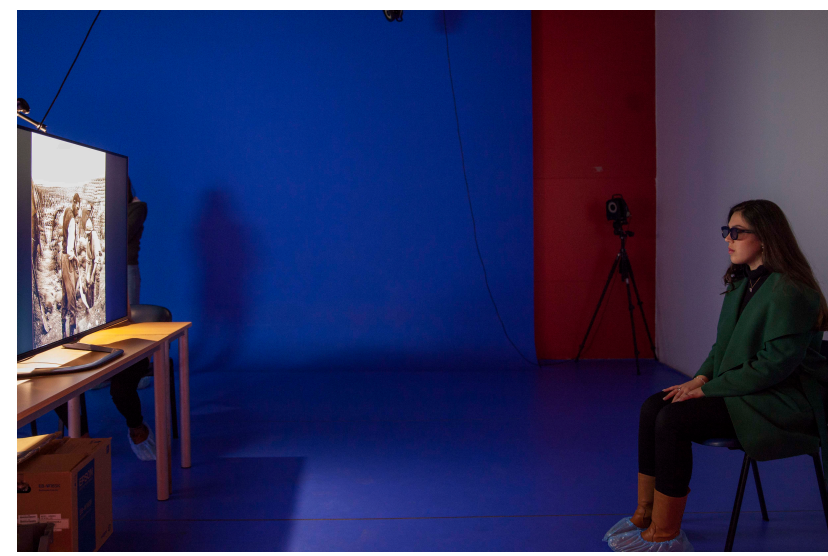

Figure 1. TV3D at MovLab facilities.

All visitants had the opportunity to interact approximately 1 minute with each image/display. After the experience they completed a questionnaire with questions designed specifically for the purpose of this study and the Self-Assessment Manikin (SAM), an established instrument in psychology to assess the 
affective experience through pleasure, arousal and dominance after the exposure to these displays. This instrument is used to measure emotional experience in a given situation involving: 1) emotional valence (pleasure) that may be assessed in an ordinal scale between positive or negative valence, 2) emotional response (arousal - the perception of activation) which is also a component of emotions, the activation of the body through different responses, and 3) dominance, which describes and additional scale to measure the involvement of the individual in the presented stimuli [5]. The responses to each of these scales varied from 1 to 9 (i.e. 1 indicating negative valence or lower activation/dominance to 9 indicating positive valence, higher activation/dominance).

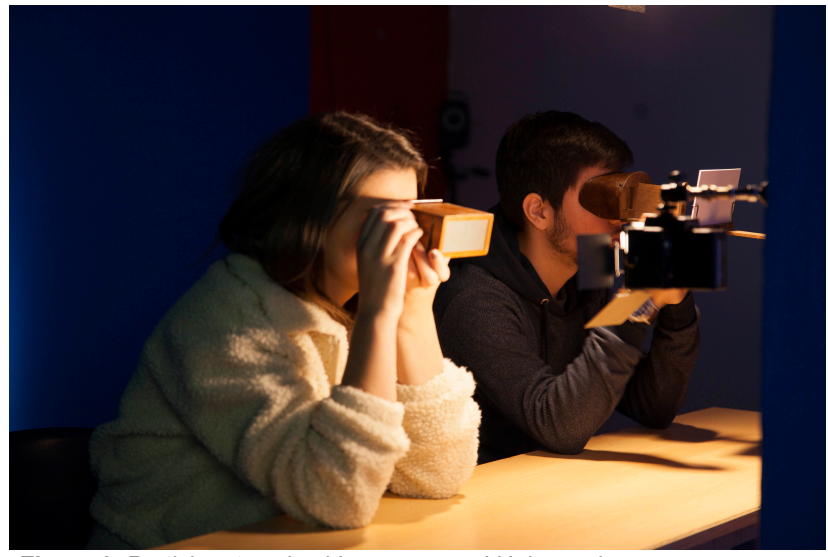

Figure 2. Participants using Verascope and Holmes viewers

The questionnaire consisted in a first section addressing socio-demographic variables as gender, age, schooling, and level of experience with computers, videogames and with the devices that were used in the study. The second section consisted of the SAM scales for each device and the third part of questions related to positive effects of exposure (e.g. immersion, interaction, etc.) and negative effects of exposure (e.g. nausea or cyber-sickness). This questionnaire was completed in a parallel room to the main room. The exposure to the stereo devices was conducted in the main room, an environment with low light conditions and specific point lights to each stereo display.

Due to vision changes/pathologies in some students (44\%), the observers had an important role to guarantee that all the visitants could absorb the $3 \mathrm{~d}$ perception. In some moments, observers decided to increase the time period of the experience to slow down the pace and avoid any undesired pressure on students. It is worth noting that despite the high proportion of vision changes/pathologies, all these participants had correctedto-normal vision.

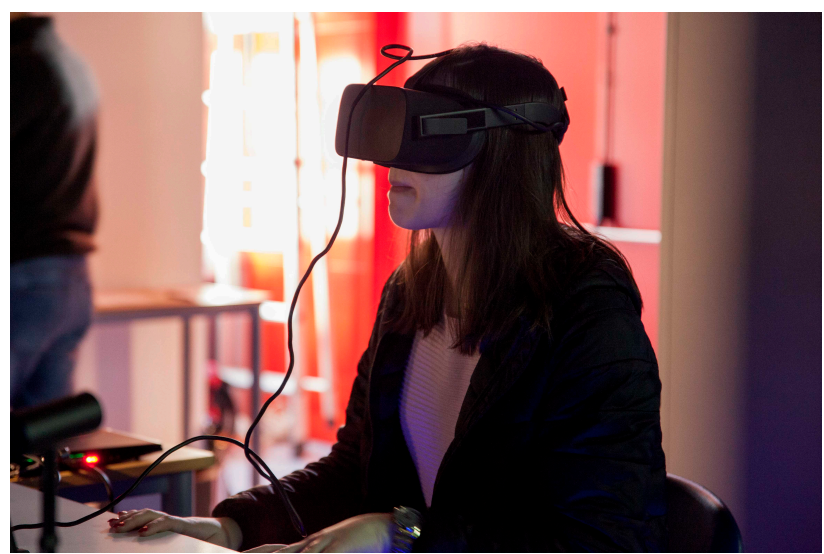

Figure 3. Participant interacting with Oculus Rift
The stereoscopic displays used were a wooden Verascope, a wooden Holmes stereoscope, a TV3D passive system, anaglyph projection (HD projector) and a VR system (Oculus Rift V2). The visitants receive a brief about the action and start to use each display individually. All the displays were interacted directly, without any intro or screen interface, however the visitants could walk in a limited space in the areas for the anaglyph projection and TV screen.

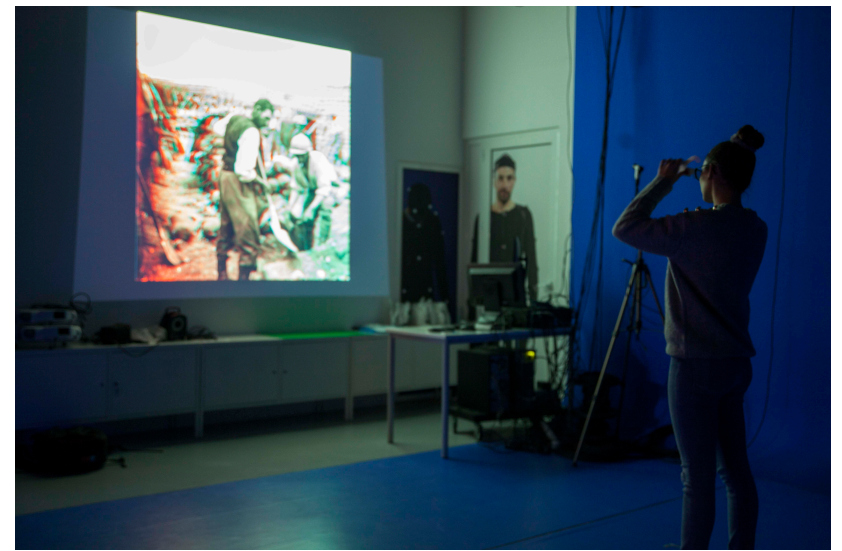

Figure 4. Participant at the anaglyph projection

In order to compare the displays, the original stereo pair was scanned in high quality ( $1200 \mathrm{dpi}$ for a $4,5 \times 12 \mathrm{~cm}$ original) to be reproduced digitally for anaglyph, VR and 3dTV (passive system in HD format 1080P) and printed into a stereocard (for Holmes Viewer). The digital conversion of analogue stereo cards demands some image manipulations in order to replicate the relief effect [5]. As the scope of this project was to reproduce the original image in several formats, the digital manipulation for the stereo viewing was limited to image size, reframing and avoiding unnecessary retouching, such as Photoshop airbrushing, that could erode the original information. In figure 6 is presented in red the areas that were cropped to create the anaglyph image, the side-by-side stereo pair and the left and right images for VR interaction.
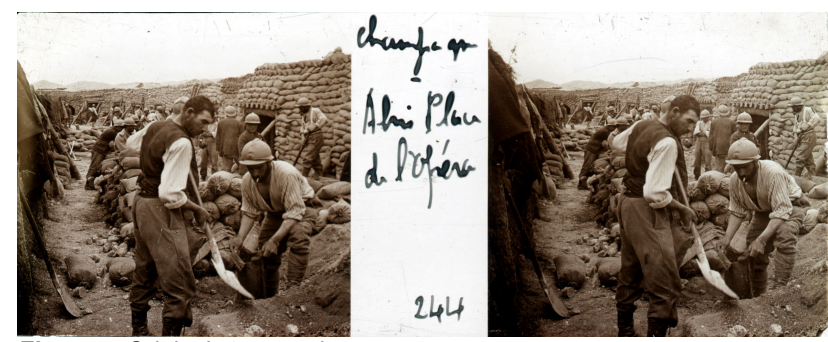

Figure 5. Original stereo pair.
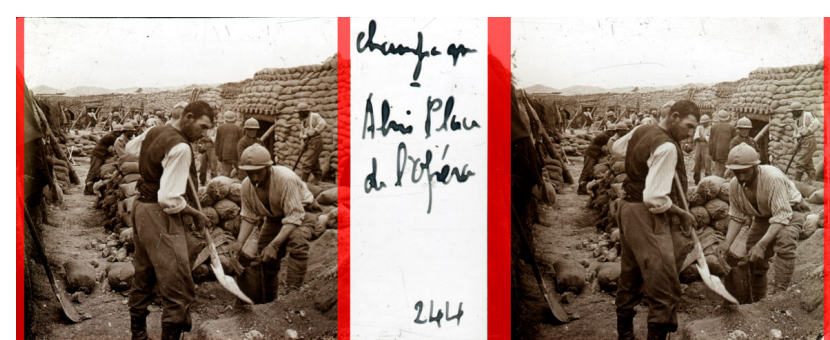

Figure 6. Reframing area for stereo digital conversion.

\section{Experiment description}

This study involved 134 university students (56\% women) aged between (18-67 years-old), mostly of Portuguese nationality $(83 \%)$ and single $(85 \%)$ in terms of civil status. Most 
participants were from psychology course $(\mathrm{n}=51 ; 40 \%)$ and videogames $(n=42 ; 33 \%)$. The remaining were from other less representative courses $(\mathrm{n}=35 ; 27 \%)$.

The fact that museums are open to a wide public, we did not place and particular exclusion criteria. In order to be included in the study, the participants had to be undergraduate students. One exclusion criterion was to have vision problems without correction that would affect stereoscopic vision. The participants were informed about the stereoscopic analysis and, in case of any vision handicap, they had to state that on the questionnaire, but all the involved participants reported normal or corrected-to-normal vision.

The exposure to each device was conducted in a random order to minimize possible order effects in the exposure. The participants were instructed about how to interact with the different displays, and in some cases, helped by the observers during the test session.

The description of this sample was done for the level of experience with computers, videogames and TV. Moreover, we also asked whether they knew each of the studied devices. The descriptive analysis to each of these variables showed that most participants reported expert levels of experience with computers $(45 \%)$. The sample reported that play occasionally $(35 \%)$ but watch TV more frequently (51\%), as for instance watching movies and series. Regarding knowledge of the devices, most participants did not know the Holmes stereo $(82 \%)$ and the Verascope $(81 \%)$. However, they were more familiar with the remaining devices, namely the Anaglyph (68\%), the TV 3D (75\%) and the Oculus Rift (65\%).

\section{Results}

The objective of the statistical analyses was to study the exposure to each of these devices at the level of emotional experience as assessed using the questionnaires developed for the purpose of this study and through the SAM scales for pleasure, arousal and dominance. These data were depicted first for the mean scores in each of these variables and then for the comparisons between these scores for each device to understand whether there were statistical differences between these scores on each device used. Significant results were considered for a .05 alpha level.

The analysis conducted for the SAM scales showed that pleasure dimension was higher for the Verascope (mean score (M) 7.02; standard deviation (SD) 1.90) and the TV 3D (M = $7.02 ; \mathrm{SD}=1.81$ ), whereas the lowest results were obtained for the Anaglyph stereo $(\mathrm{M}=5.17 ; \mathrm{SD}=2.34)$. These differences were statistically significant through the Analysis of Variance (ANOVA)

Regarding the arousal dimension, the results were in the same direction than the previous for pleasure, showing higher activations for the Verascope $(\mathrm{M}=6.60 ; \mathrm{SD}=2.00)$ and the TV $3 \mathrm{D}(\mathrm{M}=6.70 ; \mathrm{SD}=1.88)$. The lowest activation scores were for the Holmes $(\mathrm{M}=5.36$; $\mathrm{SD}=2.10)$ and the Anaglyph stereo $(\mathrm{M}$ $=5.38 ; \mathrm{SD}=2.26)$. These differences were also statistically significant through the ANOVA.

As for dominance in the SAM, the results showed that dominance were higher and very similar between the Verascope $(\mathrm{M}=6.72 ; \mathrm{SD}=2.00)$ and the TV $3 \mathrm{D}(\mathrm{M}=6.71 ; \mathrm{SD}=2.00)$. The lowest results in dominance were also obtained for the Holmes $(\mathrm{M}=5.50 ; \mathrm{SD}=2.39)$ and the Anaglyph stereo $(\mathrm{M}=$ 5.46; $\mathrm{SD}=2.42$ ). These differences were statistically significant through the ANOVA. See Chart 1 for the mean scores on each SAM dimensions.

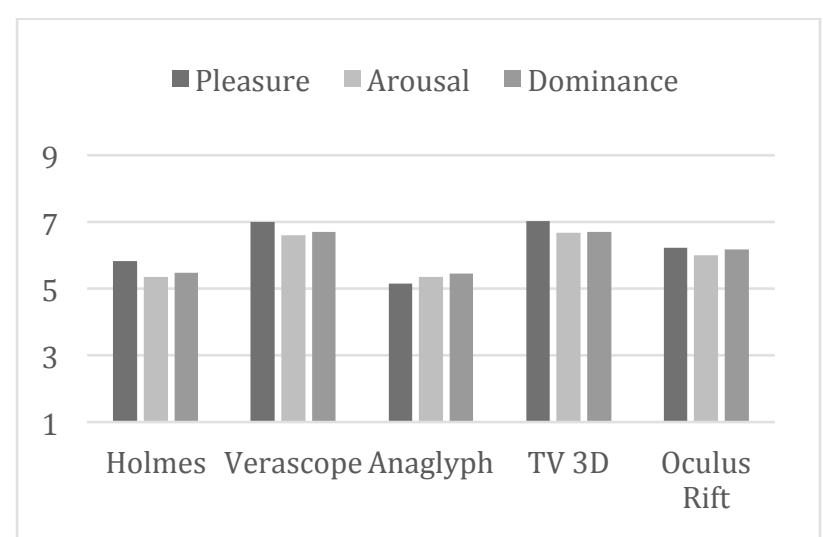

Chart 1. Mean scores on the SAM scales for each device.

Regarding the positive effects related to the exposure, the impressiveness and immersive effects of the exposure were differently evaluated by videogames and psychology students. These results were calculated with Chi-square tests. Most students of videogames reported the Verascope as the most impressive $(n=42)$ and that offers higher image definition $(n=$ 45). On the other hand, most students considered the Oculus Rift as the best immersive device $(\mathrm{n}=53)$, whereas the TV 3D was considered as the most comfortable in terms of ocular effects ( $\mathrm{n}$ $=50)$ and the device with better 3D perspective $(n=48)$. These differences between devices in each of these variables were statistically significant according to Chi-square tests.

Regarding the negative effects, interaction issues were reported for Holmes in most students $(n=59)$ when compared to the other devices. These results were also statistically significant through Chi-square tests. The level of negative ocular effects was also assessed using an ordinal response scale of 5 points ( 1 - very low to 5 - very high) in each of the devices. The results showed that there were more students reporting very high negative effects in the Anaglyph stereo $(n=29)$ than on the other devices (Chart 2).

\section{Limitations}

Any study could present several limitations, and this one is no exception. First, our measured is based mainly a young target (university students) and the info was acquired in a limited period of time in university facilities. Most of the students had a strong familiarity using the technologies presented; they were fully surprised by the quality of "old media technologies"; and the evaluation was made in a group of 5 persons. Some displays, like Verascope or Holmes, offer more struggle to get into the 3D relief, so it is always possible that some of the students could felt pressure and pass to another display without seeing the 3D effect and without telling the supervisors. As we mentioned before, the monitoring of the observers had apparently reduced the impact of this possible issue.

The technology used is updated, similar to the daily installations in worldwide museums, but this environment does not reproduce exactly the experience of visiting a museum. 


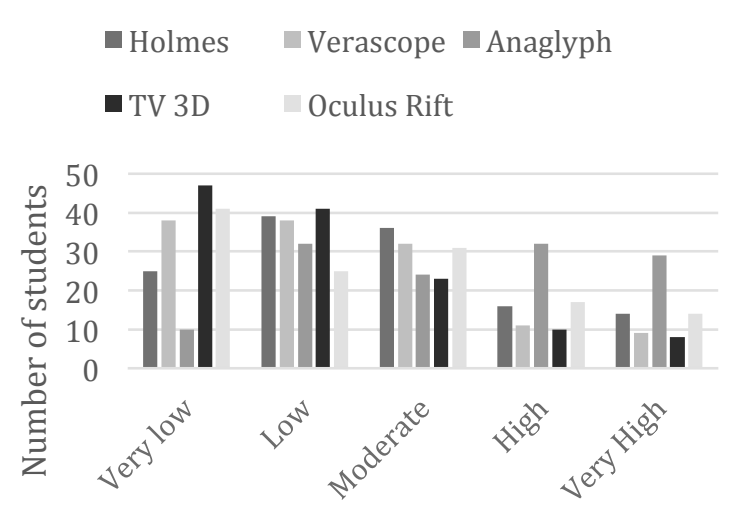

Chart 2. Number of participants reporting negative effects for each device

\section{Conclusions}

The purpose of this study was to determine which digital stereo viewing device is the best suitable for viewing analogue stereo pairs, concerning the recreation of haptic effect or Aweeffect described by users when viewing stereoscopic images in both Holmes and Verascope viewers.

The $3 \mathrm{dTV}$ seems to be the device that better reproduces the analogue experience of viewing stereo photography (better than $3 \mathrm{~d}$ projection, anaglyph or the VR device), it stands as the more suitable for the recreation of the awe-effect, an essential part of the original experience of the viewing of a stereo pair, referred by the majority of the answers as the closest to the experience of the Verascope. The Verascope viewer stands as the preferential device for a majority of the subjects in all the emotional areas pleasure, arousal and dominance. This was regarded as a surprise, since this $19^{\text {th }}$ century apparatus is quite modest when placed side by side with VR helmets and 3d TV's!

On the other hand, it came without surprise that the anaglyph experience was the one that presented the strongest negative results. Gender differences were not noted in this study.

\section{Main guidelines for digitization of stereo archives (these guidelines are only for stereo viewing purposes):}

The original images should be scanned at a resolution that allows their presentation in a HD TV, such as the one used during the study. This means that each of the images that compose the stereo pair should have at least 1920 pixels in the largest side. The resolution for the scanning depends on the size of the original, but for a standard size stereo card $(9 \mathrm{x} 18 \mathrm{~cm})$ we would recommend $800 \mathrm{dpi}$ as a basis, but $1200 \mathrm{dpi}$ would give a better definition when downsizing for the $3 \mathrm{dTV}$ file each of the individual images of the stereo pair. We are only addressing the display of the stereo image, full size in a HD $3 \mathrm{dTV}$ without zooming in. If another destination for the image is intended another scanning guidelines should be used. But, as any researcher in stereo photography knows, zooming in stereo is not a possibility.

The originals should be scanned in RGB mode (both color and black and white photographs), and the scanner should be color profiled using one of the available targets in the market; we used the Kodak IT8 target.

When scanning is not possible, due to the deterioration, non-flatness (or any other case) of the original, a photo reproduction must be done using a reproduction table (such as the Manfrotto System 750 Repro Copy Stand with Table), controlled lighting, a DSLR camera (we used the Canon EOS 5d
MkII) and, of course, a lens (we used the canon 100mm macro 2.8 lens). A color profile should be produced using a color target. We used the x-rite colorchecker Passport.

Both images that constitute the stereo pair should be cropped to their matching visual content, as showed in this article in figure 1 and 2.

Dust and scratches should be removed only when they interfere with the stereo experience, (by creating ghostly parts of the image). Otherwise they should be left at the images as witnesses of their origin and as surface proofs of the past.

The file should be saved as a JPEG with images side by side as shown in the picture. This allows viewing in the large majority of 3dTV's.

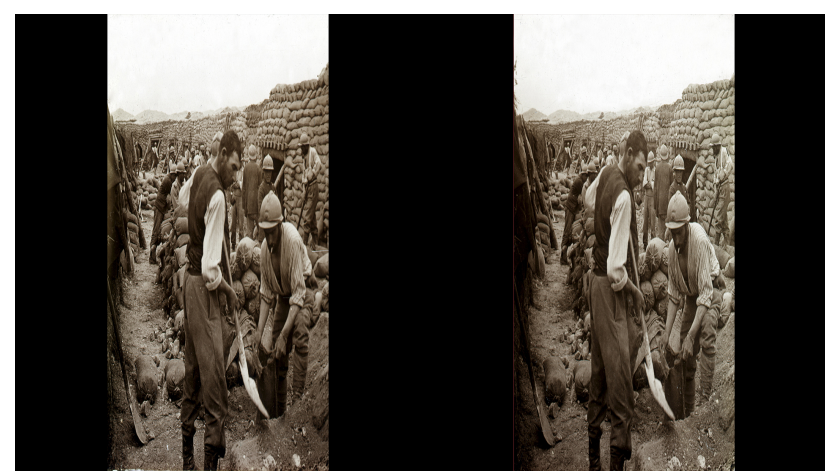

Figure 7. Side-by-side stereo image

All the material information about the original stereo pair should be presented to researchers whether at online research sites or physical research facilities. Here the researcher should have access to a description of the original referring all the standard information (i.e. author, previous owner, collection, photographic technique, date), the digital file of the original stereo pair, including borders, and the digital file of the back of the card whenever it contains additional information.

\section{References}

[1] Révèsz, G. (1950), Psychology and Art of the Blind, Longmans, Green and Co. Ltd.

[2] May, Brian (2013) Diableries, Stereoscopic Adventures in Hell in Brian May, Denis Pellerin and Paula Fleming (Org.). London: The London Stereoscopic Company.

[3] Gurevich, Leon (2013) The stereoscopic attraction: Threedimensional imaging and the spectacular paradigm 1850-2013; Convergence: The International Journal of Research into New Media Technologies. 19: 396.

[4,6] Luz, Filipe; Peixoto, R. (2017). The spectacle of stereoscopy: historical analysis and inputs to the creation of a digital archive of analog stereoscopic photography in Comunicação e Transformações Sociais (Vol. 2). IX Congresso Sopcom: Coimbra. Pp. 483-493 . ISBN 978-989-99840-1-1

[5] Bradley, M., \& Lang, P. (1994). Measuring emotion: The selfassessment manikin and the semantic differential. J Behav Ther \& Exp Psychiat, 2(1), 49-59.

\section{Author Biography}

Filipe Luz received his MSc and PhD in Communication Sciences by the New University of Lisbon, Filipe Costa Luz coordinates Videogames Ba and is vice-chair of Design Ba at ULHT. He lectures digital post-production for film, television, games and animation and also does research activities in projects of communication science, Design and arts. His work at MovLab - Laboratory of Interactions and Interfaces, it's an example of the cross media projects.

Jorge Oliveira is the coordinator of CTIP and senior researcher in COPELABs, where he was vice-director from 2014 until 2016. Jorge is also Assistant Professor at School of Psychology and Life Sciences (EPCV) in ULHT. He holds a PhD in Neuropsychology from Salamanca 
University. He is also the Principal Investigator in the CEPCA, the Research Unit of the EPCV/ULHT.

Rodrigo Peixoto is a Portuguese photographer, exhibiting regularly since 1999, the year in which he finished his studies at Ar.Co. This year he received a FLAD grant that allowed him to continue his studies at SVA, New York. In 2010 he completed his MFA at ESAD.CR. He is currently undertaking a $\mathrm{PhD}$ in Communication Studies. He was a Fellow of the
FCT foundation from 2015 to 2018. Since 2007 he teaches several subjects in Photography, Digital Animation and Cinema Licentiates at Universidade Lusófona of Humanities and Technologies. Since 2018 he is the director of the Photography BA at ULHT. 